\title{
Previous trauma exposure and perinatal depression
}

\author{
Carla Alves Pereira ${ }^{1}$, Ana Teresa Pereira ${ }^{2}$, Tânia Casanova ${ }^{1}$, Alba Roca $^{3}$ \\ 1Department of Psychiatry and Mental Health, Centro Hospitalar Tondela-Viseu, EPE., Viseu, Portugal \\ ${ }^{2}$ Department of Psychiatry and Mental Health, Vila Nova de Gaia/Espinho Hospital Center, EPE., Vila \\ Nova de Gaia, Portugal \\ ${ }^{3}$ Perinatal Mental Health Unit CLINIC- BCN, Hospital Clínic Universitari, Barcelona, Spain
}

\section{OBJECTIVES}

To present a non-systematic review of the literature on the relationship between antecedent trauma exposure and the depression in perinatal period.

\section{BACKGROUND AND AIMS}

Although all trauma types convey risk, experiencing abuse during childhood and increased frequency of such exposure are particular potent predictors of depression in perinatal period ${ }^{1,2}$. Investigating links between trauma and depression in perinatal period is needed given the frequency of perinatal depression (5$25 \%)^{1}$ and trauma history in pregnant women, ranging 29,3$74,1 \%^{3}$. Moreover, if the prior trauma does precipitate perinatal depression, then there would be major clinical implications given that depression in pregnancy predicts birth outcomes and that postpartum depression is one of the more robust predictors of compromised child health and development.

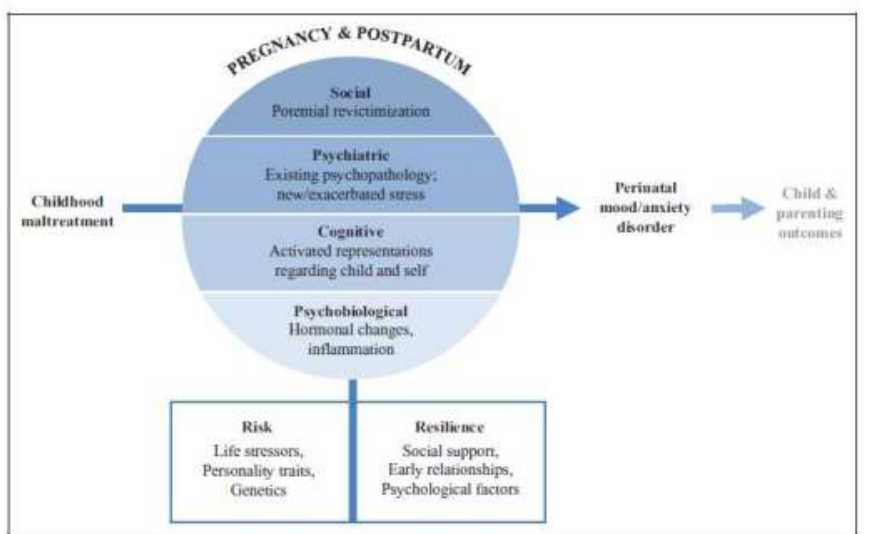

${ }^{4}$ Figure 1. Proposed conceptual model of mechanisms and moderators linking childhood maltreatment and perinatal mood and anxiety disorders.

\section{MATERIALS AND METHODS}

A literature search was conducted using the search engine Pubmed® and the keywords perinatal depression, trauma.

\section{RESULTS}

We found that trauma-exposed women appear biologically and psychological vulnerable to depressive episodes during perinatal period (Fig. 1$)^{4}$.

The finded researchs examined the risk of depression in a perinatal period as a function of antecedent trauma exposure and some of them considered the role of trauma type, timing and severity and differentiated antenatal from postnatal depression:

History of sexual abuse may have a stronger impact on mental health during pregnancy, while physical abuse may exert greater influence in the postpartum period ${ }^{4}$.

Having suffered multiple types of abuse (sexual, physical and verbal) increased three times the risk of mood and anxiety disorders during pregnancy ${ }^{2}$.

Any form of lifetime intimate partner violence, such as psychological, sexual or physical agression emerges as a one of the strongest predictor of depressive symptoms during the perinatal period, especially when it occurs during pregnancy 1 .

The coexistence then of childhood and adult abuse resulted not only in more severe depressive symptoms but also longer duration of symptoms ${ }^{1,5}$

There are also treatment implications - research has shown that a history of childhood trauma affects depression treatment response ${ }^{6}$.

\section{CONCLUSIONS}

The are evidence concerning the psychobiological relationship between trauma exposure, wich persists mostly as a pathogenic factor throughout life, and the presence of depression on perinatal period. Routine antenatal screening of depression and trauma history is required to identify women at risk and to aid in early detection and appropriate treatment of depression under these conditions.

\section{REFERENCES:}

1.Alvarez-Segura M, Garcia-Esteve L, Torres A, Plaza A, Imaz ML, Hermida Barros L, et al. (2014). Are women with a history of abuse more vulnerable to perinatal depressive symptoms? A systematic review. Arch Womens Ment Health, 17:343-357.

2.Lara MA, Navarrete L, Nieto L, Le HN. (2015). Childhood abuse increases the risk of depressive and anxiety symptoms and history of suicidal behavior in Mexican pregnant women. Revista Brasileira de Psiquiatria $37 \cdot 203-210$.

3 Robertson-Backmore E, Putnam FW, Rubinow DR, Matthieu M. Hunn JE, Putnam KT, Moynihan JA, O'Connor TG. (2013). Antecedent Trauma Exposure and Risk of Depression in the Perinatal Period. J Clin 